\title{
Die Behandlung der Meningitis und des chronischen Pyo- und Hydrozephalus durch Okzipitalinzision und Unterhornpunktion und -Drainage.
}

Von M. Westenhöfer und R. Mühsam in Berlin.

\section{Theoretischer 'Teil. \\ Von M. Westenhöfer.}

Mehrere Fälle von erfolgloser Balkenstich-Operation bei hydrozephalischen Kindern, die ich kurz vor dem Krieg zu beobachten Gelegenheit hatte, hatten mir den Gedanken nahegelegt, die Aufmerksamkeit der Chirurgen nochmals auf eine Methode der Behandlung des chronischen entzündlichen Hydrozephalus $\mathrm{zu}$ lenken, die ich in meinem Bericht über die übertragbare Genickstarre auf der 78. Naturforscherversammlung in Stuttgart vorgeschlagen habe. ${ }^{1}$ ) Die ungemein zahlreichen Fälle von Meningitis und ihren Folgen im Anschluß an Kriegsverletzungen des Kopfes bestärkten mich nur noch mehr in meinem Vorhaben, das um so mehr gerechtfertigt erscheint, da, wie im zweiten Teil gezeigt werden soll, bereits drei Fälle von Anwendung der Metho de vorliegen.

Die eitrige Hirnhautentzündung gehört bei dem in der Regel ohnmächtigen Stand der inneren Behandlung in das Gebiet des Chirurgen und sollte nach allgemeinen chirurgischen Grundsätzen behandelt werden: ubi pus, ibi evacua. Daß dies, wörtlich genommen, bei einer mehr oder weniger ausgebreiteten Eiteransammlung in den Maschen der Hirnhäute nicht durchführbar ist, ändert nichts an der Gültigkeit des Satzes, da es immerhin 'gelingt, wenigstens an einigen Stellen die Forderung $\mathrm{zu}$ erfüllen und dadurch indirekt auf entfernter liegende einzuwirken, wie es z. B. schon durch die doch sehr. weit vom Herd ansetzende Lumbalpunktion schon lange, wenn auch mit schwankendem Erfolg, geschieht.

Volle Berechtigung zur chirurgischen Behandlung, ja ihre Notwendigkeit liegt meines Erachtens vor, wenn sich in den Hirnhöhlen Eiter befindet, wenn aus einem akuten Pyozephalus ein chronischer wird und wenn es sich um einen Hydrozephalus handelt, der auf entzündlicher Grundlage entstanden ist.

Denn mit der Eiterentfernung wird überhaupt die erste Vorbedingung zur Heilung geschaffen. Da es sich außerdem noch um vorgebildete Höhlen handelt, aus deren Wand (Adergeflechte) die Eiterung durch Fortbestehen der entzündlichen Ursache auch nach Entleerung des Eiters weiter vor sich gehen kann, so muß die Methode so gestaltet werden, daß auch eine Berieselung und ergiebige Durchspülung der Höhlen erfolgen kann. Nie kann dies erreicht werden durch einfache Lumbalpunktion einerseits oder durch Balkenstich anderseits. Auch die Punktion des Hinterhorns läßt einen Hauptherd der Eiterabsonderung, nämlich die Adergeflechte des Unterhorns, unbeeinflußt. Der Grund, warum diese Methoden so oft versagen, liegt wohl hauptsächlich darin, daß die Kammer- und Hörnerhohlräume des Gehirns eine in verschiedenen Ebenen im Zickzack auf- und abwärts steigende unregelmäßige Linie bilden, deren tiefste Stelle einerseits die Spitze des Unterhorns und anderseits das Loch Magendis darstellt (s. Fig.). Diese letztere Stelle ist noch von besonderer Wichtigkeit, da hier die Gehirnkammern mit den Lymphräumen der Spinnwebenhaut, vor allen Dingen mit der großen Kleinhirn-Rückenmarkszisterne in Verbindung stehen. Eine Operation, die die Zisterne öffnet, öffnet auch die vierte und dritte Gehimkammer.

Ich habe, um eine Entlastung der Lymphraumhaut (Arach-

1) B. kl. W. 1906 Nr. 39 u. 40 S. 1315. 
noidea) im frischen Zustand der Meningitis zu erreichen, den Einschnitt und die dauernde Drainage des Ligamentum atlantooccipitale ernpfohlen.

Diese Operation war während der oberschlesischen Genickstarre-Epidemie viermal von Hartmann in Königshütte ausgeführt worden.

Die Technik ist ganz einfach und ergibt sich aus den anatomischen Verhältnissen. Die Spaltung des Bandes und der Zisterne erfolgt bei nach vorne gebeugtem Kopfe in der Längsrichtung genau in der Mittellinie. Von den vier Fällen starben zwei, die bereits im Sterben lagen, als an ihnen die Operation vorgenommen wurde, ein Fall wurde geheilt, der yierte

starb zwei Monate nach der Operation an Hydrozephalus. Dieser letzte: Fall ist gleichzeitig ein Beweis, daß diese Operation, trotzdiem sie zwei Kammern beeinflußt, nicht geeignet ist, in jedem Fall den Hydrozephalus zu heilen.

Uebrigenss ist die Okzipitalinzision neuerdings auch von $\mathrm{Payr}$ ausgeführt wdeden; so schreibt er in Nr. 33 der M. Kl. vom 13. August 1916: „Die Eröffnung der Cisterna cerebello-medullaris, die wir bei $\mathrm{Me}$ ningiti serosa acuta traumatica mit gutem Erfolg gemacht, bei eitriger Basalmeningititis vergeblich ausführten, gewinnt für die chronischen Formen der umschriebenen serösen Meningitis die größte Bedeutung!"

Größere Aussicht, den Pyo- und Hydrozephalus zu heilen, besteht dann, wenn der operative Eingriff entweder zusammen mit der Olazipitalinzision oder allein am tiefsten Punkt des Höhlensystems, nämlich an der Spitze des Unterhorns, vorgenommen wird. Je früher die Diagnose des Pyozephalus gestellt wird, je früher der Eiter aus den Gehirnkammern entleert und die Eiterabsonderung aus den Adergeflechten, insbesondere des Unterhorns beseitigt wird, um so eher wird es gelingen, den Pyozephalus zu heilen und vor allem zu verhindern, daß ein chronischier Hydrozephalus daraus wird.

Hierzu ist nicht eine einfache Punktion genügend, sondern eine längere Zeit fortgesetzte Drainage des Unterhorns mit gleichzeitiger Durchspülung der Kammern. Als Gegenöffnung kann, wenn sich eine solche als notwendig herausstellt, die Okzipitalinzision dienen.

Die Operationsstelle ist nahezu die gleiche wie die zur Herausnahme des Gasserschen Ganglions, nur daß an Stelle des großen Lappens nur eine lochförmige Anbohrung des Schläfenbeins notwendig ist. Die Stelle der Trepanation liegt $1 \mathrm{~cm}$ oberhalb del Ansatzes des Jochbeinfortsatzes an das Schläfenbein. Wird hier der Trokar eingestochen, so liegt die Punktionsöffnung der harten Hirnhaut in dem Winkel, der von dem hinteren und vorderen Ast der A. meningea media gebildet wird, letzterem nö̈her als ersteren. Die Richtung des Stichkanals ist genau horizontal; seine länge vorn Knochen an beträgt $3-4 . \mathrm{cm}$, d. h. man disrf den Trokar zunächst nicht tiefer als $3 \mathrm{~cm}$ bei Kindern, $4 \mathrm{~lm}$ bei Erwachsenen einstoßen. Es muß ein eingeteilter Trokar benutzt werden, um die Länge zu bestimmen.

Handelt es sich darum, einen schon bestehenden chronischen Hydrozephalis zu behandeln, wobei es darauf ankommt, eine neue Infektion der Höhlen zu vermeiden, so kann das MetallDauerdrainr tenem und umgebogenem Rande versehen sein, der sich dem Knochen aulien gut anlegt, wodurch sowohl ein "Hinein- wie Hinausgleiten verhindert wird. Die äußere Wunde wird durch Nähte geschlossen, sodaß die Flüssigkeit keinen Abfluß nach außen erhält, sondern subkutan aufgesogen wird. Gegebenenfalls wird sie nach dem Vorgang von Payr in die V. jugularis abgeleitet.

\section{Klinischer Teil.}

\section{Von R. Mühsam.}

Die Punktion des Unterhorns nach Westenhöfers Vorschlag ist von mir dreimal ausgeführt worden. Was die Technik betrifft, so ist sie außerordentlich einfach. Eine Allgemeinnarkose ist nicht notwendig. Es genügt die Anwendung des Chloräthylsprays auf die Stelle, an welcher die Punktion gemacht werden soll, nämlich $1 \mathrm{~cm}$ oberhalb des Ansatzes des Jochbeinfortsatzes an das Schläfenbein. Die Durchfrierung der Haut und der tieferen Schichten $\mathrm{mu}$ ßründlich und ausgiebig sein. Dann wird, wie bei der Neiss erschen Hirnpunktion, der feine elektrische Bohrer aufgesetzt, und es werden die Weichteile und der Knochen durchbohrt. Um nach Entfernung des Bohrers den in das Schädelinnere führenden Bohrkanal mit der Nadel leicht wieder finden zu können, empfiehlt es sich jetzt, solange der Bohrer noch liegt, durch nochmalige gründliche Anspritzung mit Chloräthyl den Kanal gut zu härten. Dann wird der Bohrer zurückgezogen und die Nadel eingeführt, was ohne Schwierigkeiten geschieht. Sie wird vorsichtig, etwa $3-4 \mathrm{~cm}$ vorgeschoben, bis Flüssigkeit ausfließt und mit der Spritze angesaugt werden kann.

Bei allen drei Punktionen bin ich gut in das Unterhorn hereingekommen und habe die Flüssigkeit entfernen können.

Die zur Behandlung gelangten Fälle waren an sich ungünstig und geben kein Bild über die therapeutische Leistung des Verfahrens. Die Mitteilungen sollen auch nur seine technische Ausführbarkeit beweisen und zu einer Naehprüfung auffordern.

Es seien kurz die klinischen Daten, soweit sie zum Verständnis der einzelnen Fälle notwendig sind, mitgeteilt.

Fall 1. Martha L., 17 Jahre alt, aufgenommen am 9. August 1906, entlassen am 12. August 1907. Hereditäre und familiäre A namnese ohne Besonderheiten. Mit 13 Jahren Masern, Diphtherie und Scharlach. Menses seit zwei Jahren regelmäßig. Beginn der jetzigen Erkrankung vor acht Tagen mit Kopfschmerzen, Erbrechen, Leibschmerzen und Unsicherheit des Ganges.

Befund: Grazil gebautes Mädchen. Innere Organe: ohne Besonderheiten. Leib: flach, nicht schmerzhaft. Sensorium: frei. Ganz leichte Fazialislähmung. Zunge weicht nach rechts ab. Ataxie der oberen und unteren Extremitäten. Hochgradige Schwäche derselben. Gehen unmöglich. Beim Sitzen mit geschlossenen Augen fällt Patientin nach hinten. Herabsetzung der Berührungs- und Schmerzempfindlichkeit sowie des Wärme- und Kältegefühls an der rechten unteren Extremität mit Ausnahme der rechten Fußsohle, sonst Sensibilität intakt. Blasenund Mastdarmfunktion intakt. Patellarreflexe erloschen. Kornealreflexe herabgesetzt. Linke Pupille reagiert träge, rechte starr. Rechte Pupille weiter als die linke. Links Neuritis optica, rechts Stauungspapille. Patellarreflexe aufgehoben. Bei Beklopfen der Achillessehnen. keine Zuckung. - Fs besteht Kopfschmerz des ganzen Hinterhauptes ohne bestimmte Lokalisation.

19. August. Erbrechen, starke Kopfsehmerzen, Sensibilität auch links herabgesetzt.

15. September. Zunehmende Beschwerden. Viel Erbrechen. Sensibilität recht 3 dauernd, links zeitweise herabgesetzt.

1. Oktober. Weitere Verschlimmerung, intensiver Kopfschmerz, schlechte Nahrungsaufnahme, beiderseits Areflexie der Kornea, zunehmende Fazialisparese rechts, hochgradige Ataxie der oberen und unteren Extremitäten. Patellarreflexe beiderzeits erloschen, Bauchdeckenreflexe erhalten. Kein Babinski, kein Oppenheim. Augenbefund wie bisher.

Dieser, durch Herrn Dr. Leo Jacobsohn aufgenommene Befund wurde im wesentlichen durch Herrn Prof. O p penh eịm bestätigt, welcher sein Urteil dahin zusammenfaßte: ".. daß eз sich um das Bild einer endokraniellen Neubildung handle. Vieles spricht für die rechte hintere Schädelgrube, doch sind die Erscheinungen durch die Annahme eines umschriebenen Tumors kaum zu erklären. Wenn ein begleitender Hydrozephalus auch das Schwinden der Sehnenphänomene im Bein erklärlich machen würde, so erklärt er doch nicht die doppelseitige Sensibilitätsstörung. Ueberhaupt ist das ganze Bild der Anästhesie nicht mit der Annahme eines zerebellaren Tumors in Einklang zu bringen. Ich halte es daher für wahrscheinlicher, daß es sich entweder um multiple zerebellare Neubildungen handelt, oder eine diffuse meningeale Sarkomatose vorliegt, und empfehle die Untersuchung des Spinalpunktats, halte aber auch eine Neissersche Punktion im Gebiet der rechten Zerebellarhälfte für berechtigt.". 
9. November. Lumbalpunktion. Minimaler Druck. Untersuchung des Punktats ergibt nichts Besonderes, keine Rundzellen.

12. November. Neissersche Gehirnpunktion - wegen großer Unruhe der Patientin in Chloroformnarkose - an mehreren Stellen des Gehirns und Kleinhirns. Kleine Partikelchen erweisen sich mikroskopisch als Hirn- bzw. Kleinhirnsubstanz.

Im Anschlu $B$ daran wird das linke Unterhorn punktiert. Dieses 'gelingt ganz leicht. $15 \mathrm{ecm}$ Liquor werden entleert.

14. November. Befinden hat sich nach der Punktion wesentlich gebessert, der Appetit gesteigert, wenig Erbrechen, Schlaf besser. Die Besserung nach dieser Punktion macht weitere Fortschritte, auch der objektive Befund ändert sich recht erheblich.

10. Dezember. Pupillen gleich weit, Reaktion prompt. Rechts geringe Atrophie, links keine deutliche Neuritis mehr. Kornealreflexe erhalten. Leichte Parese beider Arme, doch geringer als früher. Lagegefühl rechts aufgehoben,: links vorhanden. Astereognosis der rechten Hand; auch an den Beinen sind die Erscheinungen zurückgegangen. Patellarreflexe fehlen. Beim Aufsetzen kein Schwanken.

Die Besserung schreitet weiter fort, soda B Patientin bereits am 11. April keinen Schwindel, kein Erbrechen, keine Kopfschmerzen mehr hat. Die motorische Kraft der Extremitäten war nahezu regelrecht. Geringe Ataxie der Beine, verlangsamte Leitung für Nadelstiche mit Nachempfindung. Oberschenkel in geringem Grade hyperästhetisch, ebenso der Oberkörper. Am Oberschenkel geringe, an den Unterschenkeln ausgesprochene Hypästhesie, am Oberkörper Hyperästhesie. An den Extremitäten Empfindung für Wärme und Kälte herabgesetzt. Spontane Zuckungen mit Schmerzempfindung beider Unterschenkel.

Auch weiterhin besserte sich der Zustand der Patientin so, daß nach der Jacobsohnschen Publikation ${ }^{1}$ ) über die Patientin aus der Oppenheimschen Poliklinik folgender Bericht einging:

Es hat sich wahrscheinlich um eine akute disseminierende Enzephalomyelitis mit vorwiegender Beteiligung des Zerebellums, der Optizi und der Hinterstränge gehandelt. Der Prozeß ist größtenteils zurückgegangen. Trotz der bestehenden tabesähnlichen Symptomatologie ist ein Uebergang in multiple Sklerose wahrscheinlich. Daß auch ursprünglich peripherische neuritische Veränderungen eine Rolle geşpielt haben, ohne im Vordergrunde zu stehen, ist zuzugeben.

Zwei Jahre später, also drei Jahre nach Beginn der Erkrankung, ist die Patientin im Krankenhause Moabit nachuntersucht worden Sie fühlte sich völlig wohl und klagte nur noch über Schwäche im rechten Arm. Die letzte Untersuchung in der Poliklinik von Herrn Prof. Op penheim ergab, daß der Prozeß keine weiteren Fortschritte gemacht hatte. Man kann also von einer. Heilung mit Defekt sprechen.

In diesem neurologischen, außerordentlich komplizierten Krankheitsbilde ist eine auffallende Besserung des Zustandes nach der einmaligen Entleerung von $15 \mathrm{ccm}$ Inhalt aus dem linken Unterhorn zu verzeichnen gewesen.

Es soll dahingestellt bleiben, ob die Besserung sich nur zufällig an die Punktion anschloß, oder ob die Punktion sie einleitete. Auffallend ist jedenfalls, daß in dem fortschreitenden Verlauf der Erkrankung unmittelbar nach der Punktion ein Stillstand beobachtet wurde und sichtliche Zeichen der Besserung eintraten, sodaß man immerhin berechtigt ist, einen therapeutischen Einfluß der Punktion hier anzunehmen.

Technisch war sie leicht und einfach, nur mußte in diesem Falle die Allgemeinnarkose wegen der großen Empfindlichkeit der Patientin angewendet werden, welche sonst für die Punktion des Unterhorns, wie auch für die Neissersche Hirnpunktion, nicht notwendig ist.

Die beiden anderen Fälle von Punktionen des Unterhorns sind bei chronischem Hydrozephalus nach Meningitis ausgeführt worden.

Im ersteren Falle erübrigt sich eine genauere Mitteilung der Krankheitsgeschichte. E seien nur kurz die -wichtigsten Daten mitgeteilt:

Otto B., 17 Jahre alt, aûfgenommen am 26. Juli, gestorben am 30. September 1907. Von März 1907 an wegen Genickstarre im Krankenhause gelegen. Vier Wochen später traten Schwindel, Kopfschmerzen, Erbrechen ein; zeitweise Flimmern vor den Augen und Doppeltsehen Patient hatte bei der Aufnahme gesteigerte Patellarreflexe, keine Sensibilitätsstörung; Stehen und Gehen war unmöglich; starkes Taumeln mit Schwinndelgefühlen, Hörfähigkeit links herabgesetzt; Trägheit der Pupillenreaktion, horizontaler Nystagmius; leichte Prominenz der $\mathrm{Pu}$ pillen, deren Grenzen verwaschen sind. Stauung in der Netzhaut, keine Blutung. Sehschärfe war befriedigend. Bauchdeckenreflexe fehlen beiderseits.

Am 2. August wurde eine Spinalpunktion vorgenómmen. Kein er- höhter Druck. Flüssigkeit klar. Bei häufigem Erbrechen Kopfschmerzen, Schwindel. Es wurde am 19. August die Punktion des Unterhorns rechts gemacht und $20 \mathrm{ccm}$ trüber Flüssigkeit entleert. Bakteriologisch steril. Einige Tage danach Blasen- und Mastdarmstörungen wie auch Erbrechen, die Kopfschmerzen ließen nicht nach. Deutliche Sensibilitätsstörung für Nadelstiche an beiden unteren Extremitäten und starke Ataxie war vorhanden.

24. August wurde die Punktion links wiederholt und $10 \mathrm{cem}$ entleert. Bakteriologisch steril. Nur Blutkörperchen.

Am 28. August erneute Spinalpunktion, $140 \mathrm{~mm}$ Druck. Klare Flü ̧̧sigkeit ohne Zellen.

Am 7. November zunehmende Verschlechterung, doppelseitiger Babinski angedeutet'. Patellarreflexe beiderseits gut auslösbar. Störung des Muskel- und Gelenksinnes an beiden unteren Extremitäten, völlige Parese des' rechten Beines. Linkes Ohr vollkommen taub, rechts Hörfähigkeit in Ordnung. Pupillen starr auf Liehteinfall, Nystagmus. Hochgradige Neuritis optica. Nach einer Hirnpunktion der beiden Seitenventrikel mit Entleerung von $40 \mathrm{bzw} .10 \mathrm{ccm}$ trat keine Aenderung des Zustandes ein.

Am 18. September wurde diese Punktion wiederholt, und am 30. September trat der Exitus ein. Die Obduktion, welche nur den Schädel betrifft, ergab einen Hydrozephalus geringen Grades.


àufgenómmen am 17. Juli, gestorben am 20. Áugust 1908. Patientin hatte vor einem Jahre eine Meningitis purulenta durchgemacht. Einige Wochen später wurde der Gang schwankend, es traten starke Kopfschmerzen, die sich biз zur Unerträglichkeit steigerten, und viel Erbrechen ein. Im Vordergrunde der Erscheinungen standen bei der Patientin rasende $\mathrm{K}_{\mathrm{Opfsch}} \mathrm{merzen}$.

Am 19. August Spinalpunktion. Sie ergab nur einige Tropfen Flüssigkeit, dann kam Blut. Eine Linderung trat nicht ein.

Am 20. August Punktion der Unterhörner. Erst rechts Lokalanästhesie durch Vereisung. $25 \mathrm{ccm}$ klarer, wasserheller Flüssigkeit werden angesaugt. Hierauf wird das linke Unterhorn an der entsprechenden Stelle punktiert. Es kommt nur etwas Blut. Die Punktion wird etwas höher, gleichfalls mit negativem Erfolge, d. h. es kommt etwas Blut, wiederholt. Die Punktion war rechts garnicht, links wenig schmerzhaft. Nach der Punktion wurde der Puls etwas wechselnd, manchmal aussetzend. Patientin hatte zunächst etwas Kopfschmerzen, fühlte sich dann aber wieder wohl und war vollständigklar. Etwa eineStunde nach derPunktion wird Patientin plötzlich bei einer wechselnden Pulsfrequenz von 96 bis 160 vollständig benommen, kommt dann wieder zu sich, um eine Stunde später plötzlich zu verscheiden. Die Sektion ergibt eine Blutung. Genaueres über ihre Ursaache konnte nicht ermittelt werden.

Ein therapeutischer Effekt war in den beiden letzten Fällen, bei denen es sich schwere Folgezustände von Meningitis handelt, kaum zu erwarten. Der letzte Fall beweist, daß auch die Punktion des Unterhorns ihre Gefahren durch gelegentliche Verletzungen eines Gehirngefäßes mit sich bringt. In diesem Falle ist bei der Punktion vielleicht ein Plexusgefäß verletzt worden und hat zu tödlicher Blutung geführt. Die Erscheinungen der Blutung waren nicht ganz sicher, das Befinden der Patientin nach der Punktion auch so wechselnd, daB von einem Eingriff zur Entleerung des Blutes Abstand genommen werden mußte. Auffallend ist, daß in diesem Falle auch bei der Lumbalpunktion schon Blut entleert wurde. Es ist also wohl möglich, daß hier eine besondere Neigung zu Blutungen vorlag, doch ist es auch nicht auszuschließen, daB ein Gefäß durch die Punktion verletzt worden ist.

Wenn dieses Verfahren also auch nicht als ungefährlich angesehen werden kann; so ist es doch technisch außerordentlich einfach und verdient in geeigneten Fällen Berücksichtigung. Es ist zweifellos leichter auszuführen als der Balkenstich und diesem dadurch überlegen, daß es, wo es notwendig ist, die. Höhle des Gehirns an ihrer tiefsten Stelle trifft, wo noch Eiter vorhanden sein kann, wenn an anderen Stellen die Hirn- bzw. Rückenmarksflüssigkeit bereits klar ist. Dies scheint wichtig zu sein: Denn es zeigt, daß die Entzündung und Eiterung in den Geflechten des Unterhorns bestehen bleiben kann, wenn sie anderweitig bereits abgelaufen und beendet ist. Eine Dauerdrainage müßte daher hier besonders wirksam sein, da sie die letzten Reste entzündlichen Inhalts beseitigen dürfte.

1) Ther. d. Gegenw 1909 H. 9 S. 433 . 